changed under both transformations $T_{\theta}$ and $S_{a}$. The interpretation of this fact possesses geometric interest. When an element is turned, the common conjugate direction moves in a plane whose normal has the direction established by the ratios $G_{x}: G_{y}: G_{z}$. When an element is transformed by $S_{a}$, the line of centers of the second osculating circles has this direction.

One final detail may be mentioned. When an element is slid, the characteristic direction turns about a fixed point in the plane of the element. That is, the tangents to the characteristics form a pencil. Denote the vertex of this pencil by $V$. Direct the attention upon an element at the point $P$. When this element is turned, the line of centers of the first osculating circles will pierce the plane of the element in a point $W$. It may be shown without difficulty that $P$ is the mid-point of $V$ and $W$.

Shefrield Scientific School

May, 1917

\title{
ERRATA, VOLUME 18
}

Page 73. T. H. HiLdebrandT. On a theory of linear differential equations in general analysis.

Page 79, line 25, the expression

$$
\sum_{k=1}^{n} c_{i k} y_{0 k j}(x)
$$

should be replaced by

$$
\sum_{k=1}^{n} y_{0 i k}(x) c_{k j} .
$$

